\title{
When is a mutation not a mutation: the case of the c.594-2A>C splice variant in a woman harbouring another BRCA1 mutation in trans
}

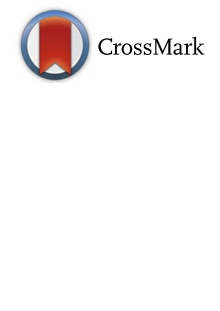

\author{
Michelle Wong-Brown ${ }^{1,2}$, Mary McPhillips ${ }^{3}$, Margaret Gleeson ${ }^{4}$, Allan D. Spigelman ${ }^{5}$ Cliff J. Meldrum³, \\ Susan Dooley ${ }^{3}$ and Rodney J. Scott ${ }^{1,2,3^{*}}$
}

\begin{abstract}
Since the identification of BRCA1 there has only ever been described two bi-allelic mutation carriers, one of whom was subsequently shown to be a mono-allelic carrier. The second patient diagnosed with two BRCA1 mutations appears to be accurate but there remain some questions about the missense variant identified in that patient.

In this report we have identified a woman who is a bi-allelic mutation carrier of BRCA1 and provide an explanation as to why this patient has a phenotype very similar to that of any mono-allelic mutation carrier. The splice variant identified in this patient appears to be associated with the up-regulation of a BRCA1 splice variant that rescues the lethality of being a double mutant.

The consequences of the findings of this report may have implications for mutation interpretation and that could serve as a model for not only BRCA1 but also for other autosomal dominant disorders that are considered as being embryonically lethal.
\end{abstract}

Keywords: BRCA1 mutations, Bi-allelic, Isoforms, Splice site

\section{Introduction}

There have been a considerable number of mutations identified in BRCA1 since its identification in 1994 [1]. During this time in excess of 15,000 BRCA1 variants have been submitted to the National Human Genome Research Institute (NHGRI)-supported Breast Cancer Information Core (http://research.nhgri.nih.gov/bic/) database. Patients harbouring a deleterious change in BRCA1 have a significantly increased risk of developing breast and or ovarian cancer [2]. Nonsense mutations represent the majority of unequivocal deleterious mutations in BRCA1 [3]. Currently, much attention has been focused on insertion/deletion mutations, missense mutations and intron-exon boundary changes since these are more difficult to classify with respect to their pathogenicity. Recent advances in the classification of these types of alteration via the development of

\footnotetext{
* Correspondence: rodney.scott@newcastle.edu.au

${ }^{1}$ Information Based Medicine Program, Hunter Medical Research Institute, Kookaburra Circuit, Newcastle, NSW 2305, Australia

${ }^{2}$ School of Biomedical Sciences and Pharmacy, Faculty of Medicine and Health, University of Newcastle, Callaghan, NSW 2308, Australia

Full list of author information is available at the end of the article
}

algorithms have significantly helped in defining pathogenicity, but it remains difficult to accurately and rapidly classify all of the variants that have been identified to date [4].

Since the identification of $B R C A 1$ much has been learned about its function, but one of the earliest findings was the paucity of bi-allelic mutations in founder populations $[5,6]$ and in mouse models of disease, where colonies of heterozygote Brca1 mice never produced any viable homozygous mutant offspring [7]. Collectively, this information implied that bi-allelic $B R C A 1$ mutations were embryonically lethal. This is in contrast to bi-allelic BRCA2 mutation carriers who do survive but invariably develop Fanconi anemia [8].

There have been two reports of patients with bi-allelic mutation in BRCA1 [6, 9]. The report by Boyd et al. [9] of a woman who was apparently a homozygous carrier of a c.2800delAA has since been shown to be a PCR artefact where preferential amplification on one allele masked the presence of the other [10]. The more recent report by Domchek et al. [6] of a developmentally delayed patient with short stature and an ovarian cancer diagnosed at 28 years of age, an unusual age even for a BRCA1 mutation carrier, appears to be the first confirmed report of a patient 
with two BRCA1 mutations in trans. One mutation in this patient is unequivocally causative, a c.2576delC change residing in exon 11 and a missense change c.5207 $\mathrm{T}>\mathrm{C}$ (Val1736Ala) located in exon 20. The Val1736Ala missense variant has been subjected to an extensive battery of tests that includes in silico analysis in conjunction with segregation and functional analysis, which clearly indicate its pathogenicity. However, there remains some questions about the pathogenicity of Val1736Ala, as logistic regression modelling suggests otherwise [11] and it is not known what, if any, residual function can be associated with the variant. This raises the possibility that the missense variant may retain function and that other factors may be involved that are not related to BRCA1 that may have led to her early demise. The more recent report by Sawyer et al. [12] highlighted a patient with the same developmental delay and diagnosis of breast cancer at 23 years of age. This patient harboured one unequivocally causative mutation, c.594_597del (p.Ser198Argfs*35) and another missense mutation on exon 18 (c.5095C > T; p.Arg1699Trp) that has previously been reported to be pathogenic and confers increased risk to breast and ovarian cancers [13]. This patient, however, was deduced to have a subtype of FA.

We herein report on a woman who developed breast cancer at an early age with no developmental delay who was referred to our laboratory for genetic testing and subsequent follow-up studies since she was shown to harbouran unequivocal BRCA1 mutation and a splice site mutation in trans, both of which are predicted to result in the absence of functional full-length wildtype BRCA1.

\section{Methods}

This study was approved by the institutional review board of the Hunter New England Health service.

\section{DNA Extraction}

DNA was extracted from peripheral whole blood by salt extraction.

\section{BRCA1 Mutation Analysis}

The entire coding sequence including the intron-exon boundaries of BRCA1 was amplified using primer sequences design in the laboratory. The PCR reactions were treated with ExoSAP (Thermo Fisher Scientific) prior to being bidirectionally sequenced with the BigDye Terminator v3.1 Cycle Sequencing Kit and capillary-electrophoretically separated using an ABI3730 DNA Analyzer automated sequencer (Applied Biosystems). Sequencing traces were analysed using Mutation Surveyor v3.24 (SoftGenetics). Mutations were described using the nomenclature guidelines of the Human Genome Variation Society (http://www.hgvs.org). The DNA sequence numbering is based on the cDNA sequences for BRCA1 (NM_007294.3).

\section{RNA Extraction}

Lymphocytes isolated from the patients were infected with Epstein-Barr virus (EBV) to establish the immortal lymphoblastoid cell lines (LCLs). These LCLs were maintained in RPMI media (Thermo Scientific) supplemented with $10 \%$ fetal calf serum (Sigma-Aldrich) in an incubator set to $37^{\circ} \mathrm{C}$ and $5 \% \mathrm{CO}_{2}$. Puromycin (InvivoGen) was added to the EBV cells to prevent RNA degradation by nonsensemediated decay (NMD).

Total RNA was isolated from cells of carrier patients using Trizol $^{\bullet}$ (Invitrogen ${ }^{\mathrm{TM}}$ ) according to the protocol provided by the manufacturer. RNA was reverse transcribed to cDNA using SuperScript ${ }^{\text {th }}$ II reverse transcriptase (Invitrogen $^{\mathrm{mt}}$ ) according to the protocol provided by the manufacturer.

\section{Sequencing of BRCA1 isoforms}

Primers were designed to flank the region of interest $\left(\right.$ Invitrogen $\left.{ }^{\mathrm{Tm}}\right)$. One forward primer $\left(5^{\prime}\right.$-TGAGAACTCT GAGGACAAAGCA-3') is located in exon 8 and the other (5'-CTGAAGATACCGTTAATAAGGCA-3') is located in exon 9. The reverse primer $\left(5^{\prime}\right.$-CCCTGATA CTTTCTGGATGC) is located in exon 11. Polymerase chain reaction (PCR) was conducted under the following cycling conditions: denaturation at $95{ }^{\circ} \mathrm{C}$ for $5 \mathrm{~min}$; $32 \mathrm{cy}$ cles of $95{ }^{\circ} \mathrm{C}$ for $30 \mathrm{~s}, 59^{\circ} \mathrm{C}$ for $40 \mathrm{~s}$ and $72{ }^{\circ} \mathrm{C}$ for 40 , and extension at $72{ }^{\circ} \mathrm{C}$ for $10 \mathrm{~min}$. The PCR products were then size-separated by electrophoresis on a $2 \%$ agarose gel.

Direct sequence analysis was performed using the BigDye $^{\circ}$ Terminator v3.1 Cycle Sequencing Kit (Applied Biosystems) and size separated by capillary-electrophoresis using an ABI3730 DNA Analyzer (Applied Biosystems). Sequencing traces were analysed using Mutation Surveyor v3.24 (SoftGenetics ${ }^{\circ}$.

\section{Real-time analysis of $B R C A 1$ isoforms}

Real-time PCR was performed using SYBR ${ }^{\circ}$ Green PCR amplification on an Applied Biosystems ABI PRISM ${ }^{\circ}$ $7900 H T$ Sequence Detection System (SDS). A $12.5 \mu \mathrm{l}$ reaction volume was set up, which included $6.25 \mu$ SYBR $^{\circ}$ Green PCR MasterMix, $0.25 \mu \mathrm{l} \mathrm{B}$-actin (housekeeping gene) or custom BRCA1 forward and reverse primers $(5 \mu \mathrm{M})$ (Table 1), and $0.625 \mu \mathrm{l}$ reverse transcription product from the patient and two controls. All reactions were run in triplicate. The reactions were incubated at $50{ }^{\circ} \mathrm{C}$ for $2 \mathrm{~min}$ and $95{ }^{\circ} \mathrm{C}$ for $10 \mathrm{~min}$, followed by $40 \mathrm{cy}$ cles of $95{ }^{\circ} \mathrm{C}$ for $15 \mathrm{~s}$ and $60{ }^{\circ} \mathrm{C}$ for $1 \mathrm{~min}$. The results were analysed using the SDS software.

\section{Results}

The patient presented with breast cancer at 30 years of age without any immediate family history (pedigree shown in Fig. 1). The tumour was defined as being estrogen, progesterone and HER2 receptor negative, and 
Table 1 Sequences of forward and reverse primers used for the detection of expression of BRCA1 isoforms by RT-PCR

\begin{tabular}{|c|c|c|}
\hline Isoform & Forward sequence & Reverse sequence \\
\hline Wildtype BRCA1 (does not cover truncation in exon 11) & 5'-GCAACTTATTGCAGTGTGGG-3' & 5'-ACAAGCAGCCTTITTTGCAG-3' \\
\hline Exon 10 deleted $(\Delta 10)$ & 5'- GCAACTTATTGCAGCTGCTTG-3' & 5'- AGCTGCACGCTTCTCAGTG-3' \\
\hline Exon 9 and 10 deleted $(\Delta 9 / 10)$ & 5'- GTCTGTCTACATTGAATTGGCTG-3' & 5'- AGCTGCACGCTTCTCAGTG-3' \\
\hline Exon $11 \mathrm{q}$ deleted $(\Delta 11 \mathrm{q})[12]$ & 5'-CCAACTCTCTAACCTTGGAACTGTG-3' & 5'-GATGACCTTTCCACTCCTGGTTC-3' \\
\hline
\end{tabular}

thus suggesting that due to her young age she was likely to have a genetic predisposition to early onset disease.

Genetic testing of DNA derived from her peripheral blood lymphocytes revealed a frameshift mutation in exon 11 of BRCA1 (c.2681_2682delAA) and a splice site change located in intron 9 that results in the absence of exon 10. This splice site change, c.594-2A > C, is predicted to be a splice site mutation that causes a frameshift, resulting in a premature stop codon). Genetic testing of the mother revealed that she carried the c.2681_2682delAA mutation and the father the c.594$2 \mathrm{~A}>\mathrm{C}$ splice site variant. The BRCA1 exon 11 mutation is clearly pathogenic and is associated with an increased risk of early onset breast and/or ovarian cancer. The presence of two potentially pathogenic mutations in trans observed in a single individual appears to be contrary to the evidence provided by animal models and founder populations where BRCA1 mutations in trans are either embryonically lethal or unreported $[6,7]$. The presence of a splice site mutation does not automatically infer that splicing is affected, so to ensure that splicing was indeed interrupted we designed a series of primers to interrogate the expression of the allele that carried the splice site variant. Primers located in exon 8 and exon 11 were used to identify which transcripts were present in the cDNA of the proband compared to a healthy control subject. The primer used in exon 11 was $5^{\prime}$ of the c.2681_2682del mutation and as such is referred to as the "wildtype" allele. The results revealed the presence of three transcripts, the "wildtype" transcript, the exon 10-deleted $(\Delta 10)$ transcript and a commonly occurring isoform with exons 9 and 10 deleted $(\Delta 9 / 10)$ transcript (Fig. 2). In the healthy control subject only the wildtype and $\Delta 9 / 10$ transcript can be identified, confirming the specificity of the exon 10 deletion in the proband.

The paternal allele carries a c.641G polymorphism in exon 10 (Gly214) which is in linkage disequilibrium with the c.594-2A > C mutation. If read-through of the splicing variant was occurring, the allele produced would lead to the translation of a functional BRCA protein. To determine whether this allele was expressed, a PCR was performed using primers which annealed to exon 9 and exon 11 ( $5^{\prime}$ of the maternal mutation). The amplified fragment would exclude the 9/10del isoform but would include exon 10 of the maternal allele as well as the paternal allele if present. If this fragment was heterozygous for the $641 \mathrm{~A}>\mathrm{G}$ polymorphism it would indicate that exon 10 of the paternal allele was present, indicating that the c.594-2A > C mutation is not fully penetrant.

Our results revealed that only the maternal wildtype allele was expressed and that no paternal allele could be detected (Fig. 2). This result confirmed that the c.594$2 \mathrm{~A}>\mathrm{C}$ did affect the expression of the paternal allele, thus the functional BRCA1 $(\Delta 9 / 10)$ trranscript must be produced from an alternatively-spliced isoform.

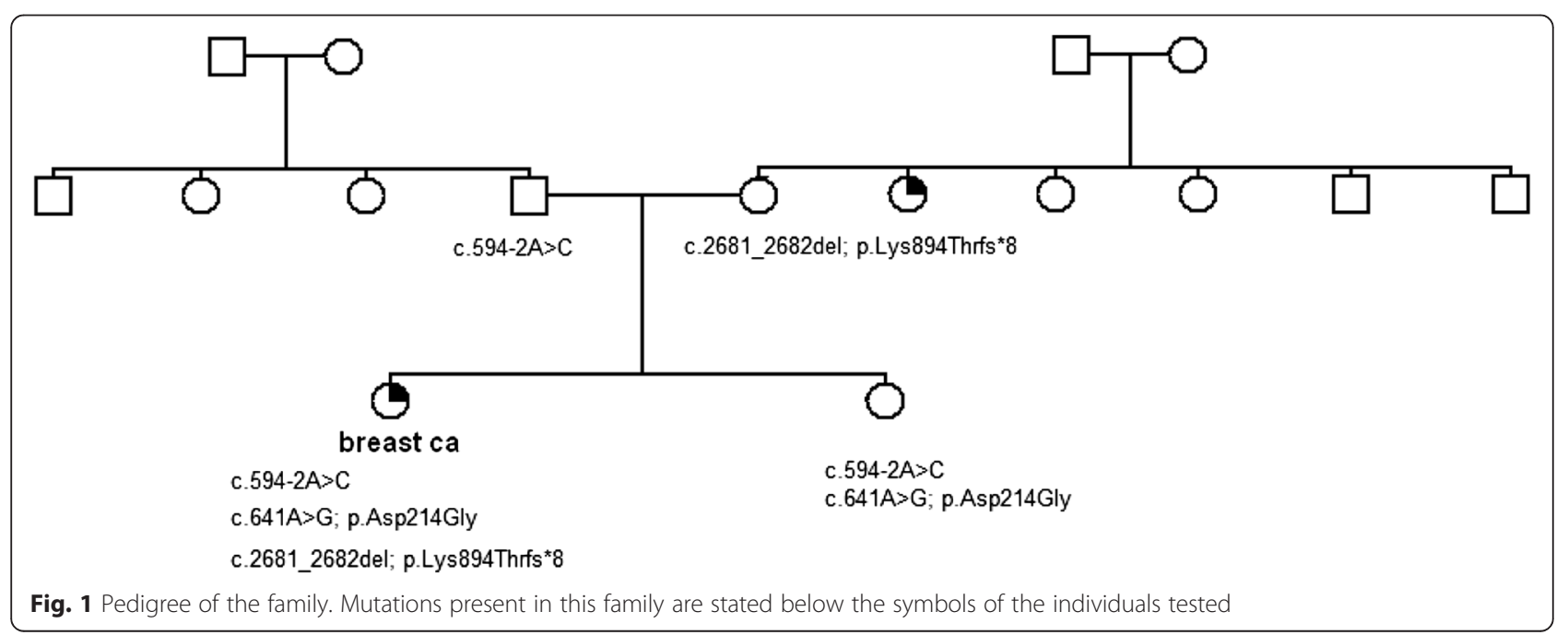




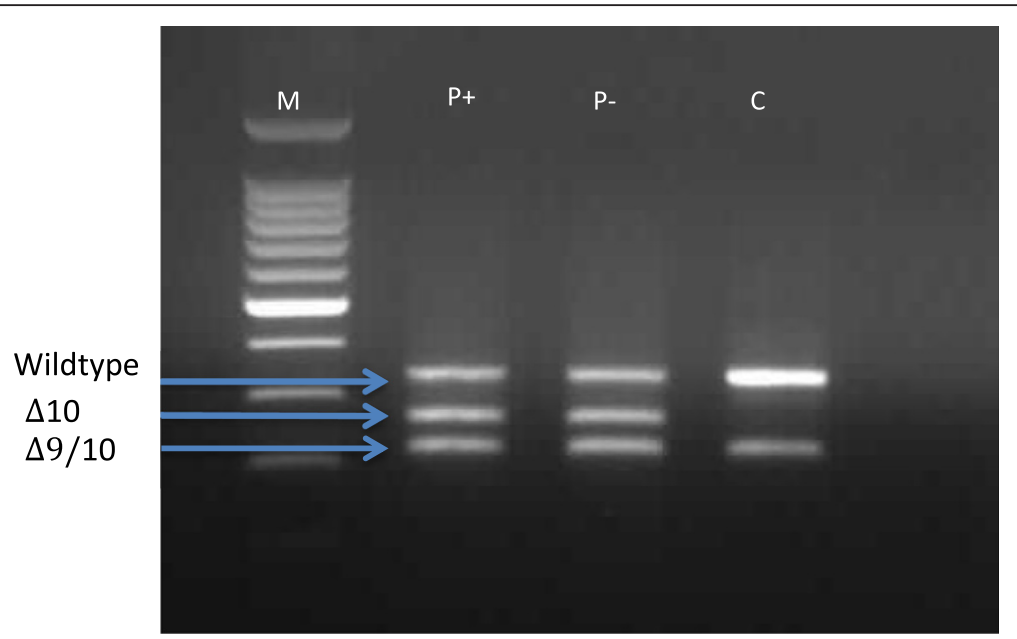

Fig. 2 Analysis of the BRCA1 mutation c.594-2 A > C by agarose gel electrophoresis. PCR products amplified using primers specific for exon 8 and exon 11 of the BRCA1 gene. $P$ is the patient sample and $C$ is the control sample. Puromycin treated $(P+)$ and puromycin non-treated $(P-)$ are indicated in the figure. $M$ is the 100 bp DNA ladder (Promega). Three different transcripts are present in the proband (P) representing a wildtype fragment, a fragment lacking exon 10 and a fragment representing the $\Delta 9 / 10$ isoform Only the wildtype fragment and the $\Delta 9 / 10$ isoform are present in the control sample (C)

Since the maternal and paternal alleles are not expressed as a result of the respective mutations they carry, the question arises as to what is occurring in the proband that has allowed her to survive and present with a phenotype that is similar, to a women harbouring a single deleterious change in BRCA1. We aimed to determine whether one or more isoforms of BRCA1 could rescue the phenotype such that a functional BRCA1 transcript was produced but not of full length (Fig. 3). For this analysis we used real-time polymerase chain reaction (RT-PCR) to quantify the levels of transcripts present in the proband and a series of control subjects (see Fig. 4). Primers were designed to specifically capture the expression levels of the $\Delta 10$ transcript, as well as the $\Delta 9 / 10$ isoform and another functional exon 11q-deleted ( $\Delta 11 \mathrm{q})$ isoform [14]. Both the $\Delta 9 / 10$ isoform and the $\Delta 11 \mathrm{q}$ isoforms are in-frame and have potential residual functional activity. We compared the results from the proband against a normal control and a second breast cancer patient who harboured a mutation in one allele that resulted in the deletion of exon 10 (c.581C > T;p.Ala194Val). The results revealed that in the control subject expression of the $\Delta 9 / 10$ isoform is detectable at approximately the same level as the wildtype BRCA1 transcript. There is also expression of the $\Delta 9 / 10$ isoform that is similar to wildtype BRCA1 expression levels in the breast cancer patient control (heterozygous mutation carrier). In both of these subjects, the $\Delta 11 \mathrm{q}$ isoform and the $\Delta 10$ isoform (specific to the proband) were not detectable. The $\Delta 9 / 10$ isoform was substantially upregulated by about 2.5 -fold relative to the levels of the BRCA1 "wildtype" transcript in the proband. The $\Delta 10$ transcript was observed to be expressed at approximately similar levels to the "wildtype" transcript, and we also showed the expression of the $\Delta 11 \mathrm{q}$ isoform. This collective observations suggest that the $\Delta 9 / 10$ isoform and the $\Delta 11 \mathrm{q}$ isoform is upregulated in the proband compared to that observed in the control subject and another breast cancer patient control.

\section{Discussion}

There is a paucity of patients harbouring BRCA1 mutations in trans, which is considered to be due to the embryonically-lethal nature of these events. Epidemiological evidence in founder populations and animal models support this view and to date only one example of a patient harbouring two mutations in trans has been reported [6]. Even though the report from Domchek et al. (2012) is comprehensive in its assessment of the missense change there remain some questions about the reported patient. Given the developmental delay, short stature and microcephaly observed in the patient reported by Domchek et al. (2012), it remains to be determined if there were other genetic alterations that could give rise to the patient's severe symptoms. And like this previous report, a more recent one by Sawyer et al. (2015) [12] showed a patient with biallelic BRCA1 mutations in trans with a similar phenotype.

Like the splice site mutation in our proband, there is a possibility that the missense Val1736Ala and the Arg1699Trp mutations possibly retain some function, as the two probands harbouring the missense and nonsense mutations in trans have a low age of cancer onset even for monoallelic BRCA1 mutation carriers [6]. Nevertheless, these patients harboured an unequivocal BRCA1 mutation and a variation of unknown functional significance that is very likely to be pathogenic. The difference between this 


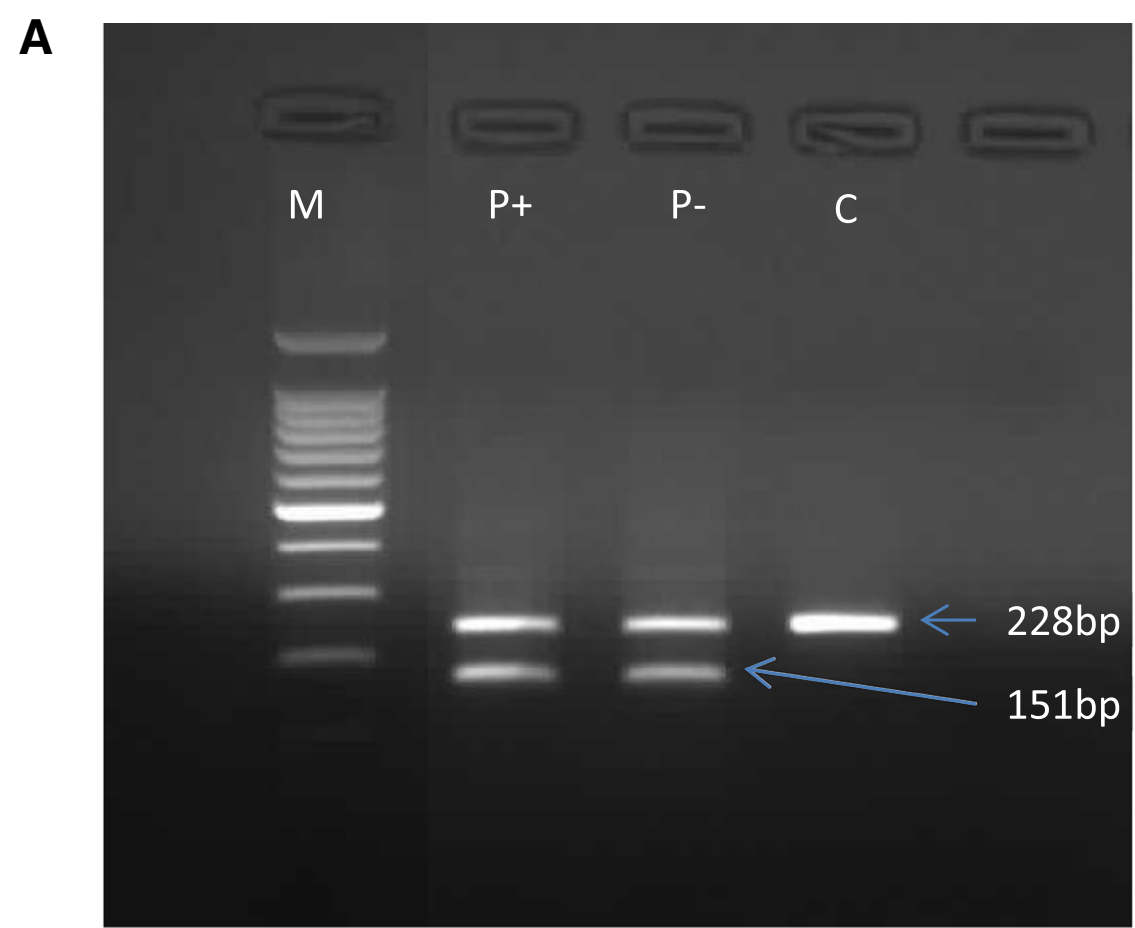

B

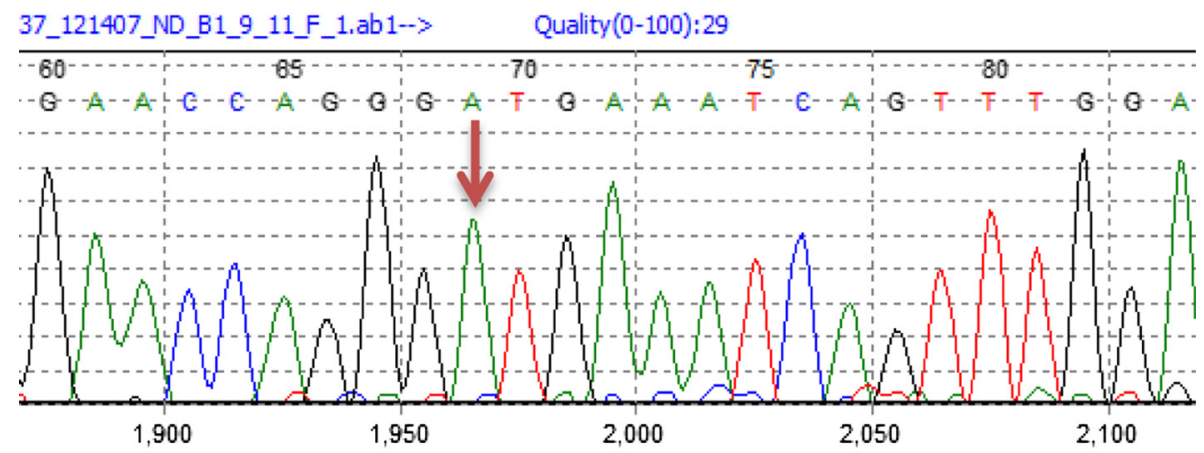

C

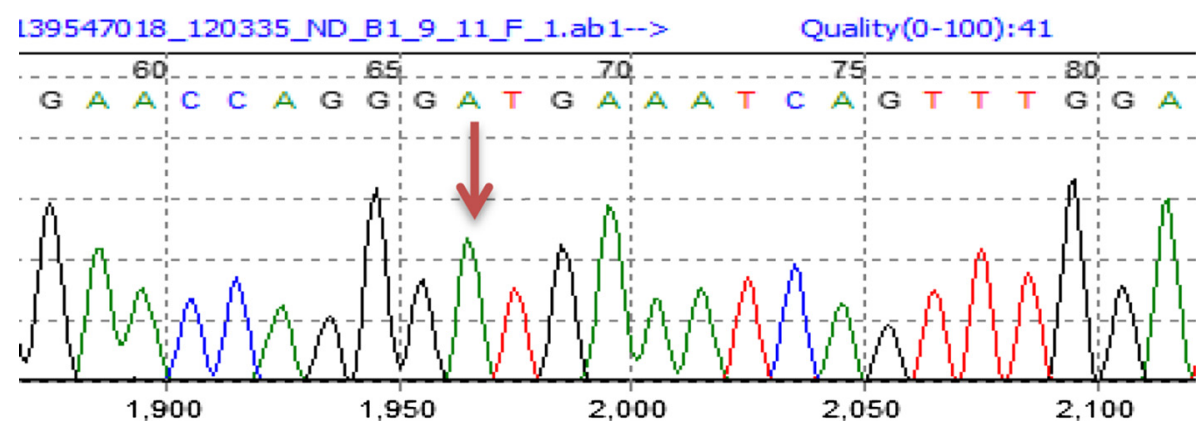

Fig. 3 Analysis of the BRCA1 variant C.641A>G. a Agarose gel showing PCR products amplified using primers specific for exon 9 and exon 11 of the BRCA1 gene. $P$ is the patient sample while $C$ is the control sample. Puromycin treated $(+)$ and puromycin non-treated (-) are indicated in the figure. $\mathrm{M}$ is the $100 \mathrm{bp}$ ladder size marker (Promega). The band at 228bp represents the fragment amplified from exon 9 to exon 11 and includes exon 10. The band at $151 \mathrm{bp}$ represents the fragment lacking exon 10. b Sequencing analysis of the 228bp band for the patient indicates the presence of the c.641A allele (arrow), but not c.641G. c Sequencing of the 228bp band for the control sample indicates the presence of the c.641A allele 


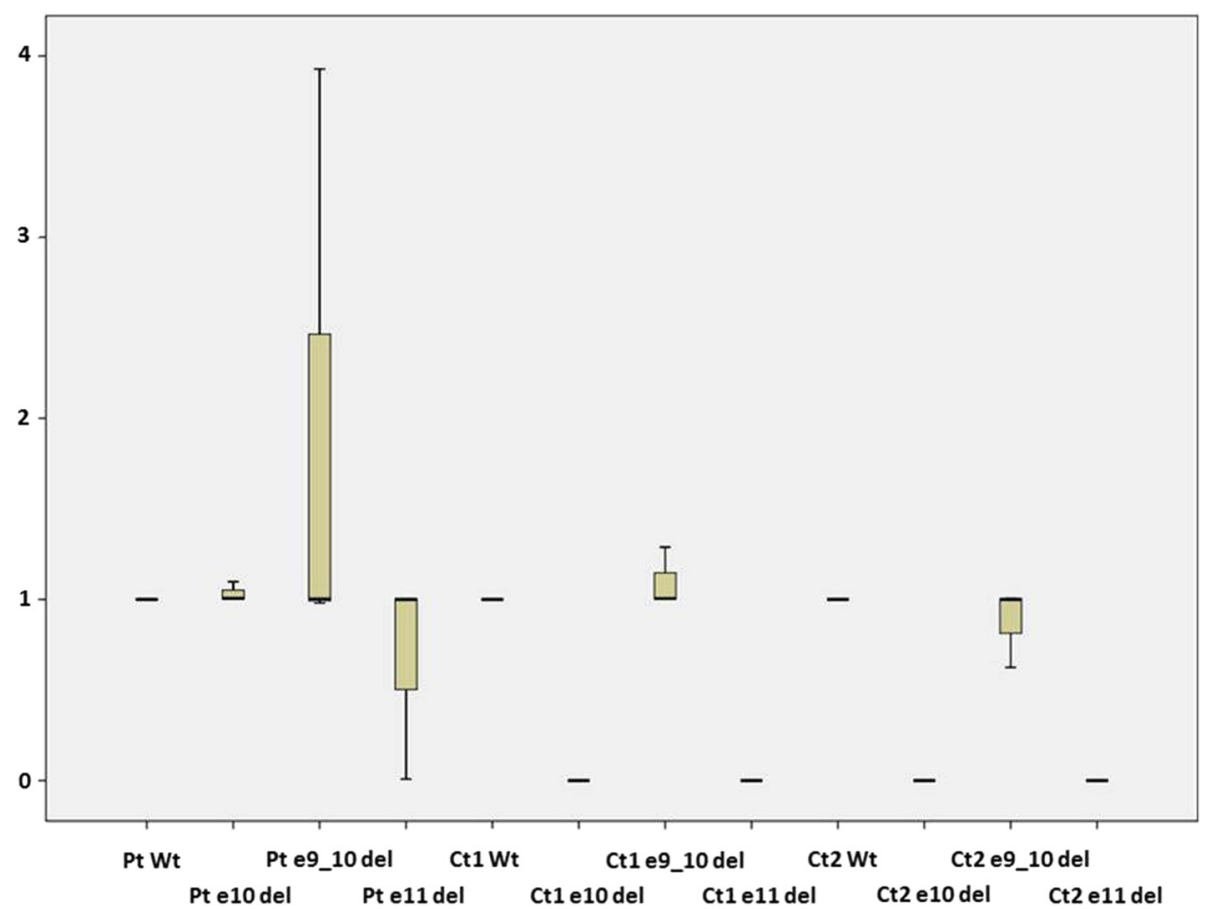

Fig. 4 Relative expression (2- $\triangle \triangle C T$ ) of each of the BRCA1 isoforms compared to the wildtype BRCA1 in patient and controls. The BRCA1 $\triangle 10$ and $\Delta 11 \mathrm{q}$ isoforms are not present in any of the two controls

patient and the one reported herein is that there appears to be a rescue of the phenotype by virtue of the fact that a functional isoform is upregulated on the allele harbouring the c.594-2A>C variant. The functional isoform is linked to the presence of the intronic splice site mutation adjacent to exon 10. This alteration results in the loss of exon 10 and concomitantly activates a known isoform that appears to be similar in function to the wildtype allele [15]. In support of this, we were fortunate enough to encounter another unrelated patient who harboured a single mutation at the 3' end of exon 9 (c.581C>T, p.Ala194Val), also predicted to result in alternate splicing. Interestingly, in this patient it was observed that the $\Delta 9 / 10$ isoform was also upregulated but not to the same extent as that observed in the proband reported herein. It has been reported that the c.594-2A>C variant is not associated with a highly-penetrant disease profile even though the allele is not expressed in persons who harbour this change [16]. It is likely that as this variant has been detected in trans with other unequivocally pathogenic mutations, such as the truncating c.2681_2682del in our patient and the BRCA1 founder variant exon13ins6kb [16], the pathogenicity of this variant has not been until now fully elucidated but has been the subject of investigation [17]. We have unequivocally shown that the BRCA1 c.594-2A $>$ C variant results in a loss of the full-length wildtype allele and the upregulation of a functional BRCA1 isoform. This is, to our knowledge, the first evidence demonstrating the functional activity of the $\Delta 9 / 10$ BRCA1 isoform. Segregation analysis of families harbouring this variant clearly indicates reduced penetrance that is at odds with expression data which unequivocally shows the absence of the allelespecific transcript [18]. In the absence of any other information this would create a conundrum in terms of what it meant to harbour this change. The data from the proband suggests there is a feedback loop associated with the splice site mutation and the expression of the $\Delta 9 / 10$ isoform that results in the rescue of the phenotype, which is consistent with the report from Dosil et al. (2010) who characterised the role of the splice variant c.591C $>\mathrm{T}$ in the generation of the $\Delta 9 / 10$ isoform [19]. Taken together, result explains the significantly reduced disease penetrance observed in mono-allelic carriers of the c.594-2A >C variant.

\section{Conclusion}

We have an example of a true biallelic mutation in $B R C A 1$, both of which significantly alter the full-length transcripts of BRCA1. However, as a result of upregulation of the $\Delta 9 / 10$ functional isoform rescue of the phenotype occurs. Without the compensation of the $\Delta 9 / 10$ isoform it is likely that either the patient would have not developed in utero or would have had severe mental retardation. This implies that the $\Delta 9 / 10$ isoform is functional and capable of orchestrating most, if not all, of the function of the wildtype allele. Acknowledgement of this finding will make genetic analysis and counselling issues more 
complex since mutations occurring in region of exons 9 and 10 of BRCA1 may not result in predicted outcomes based on sequence analysis alone.

\section{Competing interests}

The authors declare that they have no competing interests.

\section{Authors' contributions}

MW-B, MM, CJM \& SD carried out the molecular studies, participated in the sequence analysis and interpretation of the molecular data. MG \& ADS provided clinical information about the patient and other members of the patient's family. RJS coordinated the study and wrote the manuscript as well as overseeing the molecular analysis and data interpretation.

\section{Acknowledgements}

The authors would like to acknowledge Pathology North for funding the study as part of their commitment to providing comprehensive diagnostic BRCA1 \& BRCA2 testing.

\section{Author details}

'Information Based Medicine Program, Hunter Medical Research Institute, Kookaburra Circuit, Newcastle, NSW 2305, Australia. ${ }^{2}$ School of Biomedical Sciences and Pharmacy, Faculty of Medicine and Health, University of Newcastle, Callaghan, NSW 2308, Australia. ${ }^{3}$ Division of Molecular Medicine, Pathology North, John Hunter Hospital, Lookout Road, Newcastle, NSW 2305, Australia. ${ }^{4}$ Hunter Family Cancer Service, Hunter New England Health District, Newcastle, NSW 2300, Australia. ${ }^{5}$ UNSW Medicine, St. Vincent's Hospital Clinical School, Sydney, Sydney, NSW 2010, Australia.

Received: 6 August 2015 Accepted: 29 December 2015

Published online: 16 February 2016

\section{References}

1. Miki Y, Swensen J, Shattuck-Eidens D, Futreal PA, Harshman K, Tavtigian S, et al. A strong candidate for the breast and ovarian cancer susceptibility gene BRCA1. Science. 1994;266(5182):66-71.

2. Ford D, Easton DF, Stratton M, Narod S, Goldgar D, Devilee P, et al. Genetic heterogeneity and penetrance analysis of the BRCA1 and BRCA2 genes in breast cancer families. The Breast Cancer Linkage Consortium. Am J Hum Genet. 1998;62(3):676-89.

3. National Human Genome Research Institute. An Open Access On-Line Breast Cancer Mutation Data Base. 2009 January 30, 2010 May 22, 2010]; Available from: http://research.nhgri.nih.gov/bic/.

4. Spurdle AB, Healey S, Devereau A, Hogervorst FB, Monteiro AN, Nathanson $\mathrm{KL}$, et al. ENIGMA-evidence-based network for the interpretation of germline mutant alleles: an international initiative to evaluate risk and clinical significance associated with sequence variation in BRCA1 and BRCA2 genes. Hum Mutat. 2012;33(1):2-7.

5. Gorski B, Byrski T, Huzarski T, Jakubowska A, Menkiszak J, Gronwald J, et al. Founder mutations in the BRCA1 gene in Polish families with breast-ovarian cancer. Am J Hum Genet. 2000;66(6):1963-8.

6. Domchek SM, Tang J, Stopfer J, Lilli DR, Hamel N, Tischkowitz M, et al. Biallelic deleterious BRCA1 mutations in a woman with early-onset ovarian cancer. Cancer Discov. 2013:3(4):399-405.

7. Evers B, Jonkers J. Mouse models of BRCA1 and BRCA2 deficiency: past lessons, current understanding and future prospects. Oncogene. 2006; 25(43):5885-97.

8. Howlett NG, Taniguchi T, Olson S, Cox B, Waisfisz Q, De Die-Smulders C, et al. Biallelic inactivation of BRCA2 in Fanconi anemia. Science. 2002; 297(5581):606-9.

9. Boyd M, Harris F, McFarlane R, Davidson HR, Black DM. A human BRCA1 gene knockout. Nature. 1995;375(6532):541-2.

10. Kuschel B, Gayther SA, Easton DF, Ponder BA, Pharoah PD. Apparent human BRCA1 knockout caused by mispriming during polymerase chain reaction: implications for genetic testing. Genes Chromosomes Cancer. 2001;31(1):96-8

11. Easton DF, Deffenbaugh AM, Pruss D, Frye C, Wenstrup RJ, Allen-Brady K, et al. A systematic genetic assessment of 1,433 sequence variants of unknown clinical significance in the BRCA1 and BRCA2 breast cancerpredisposition genes. Am J Hum Genet. 2007;81(5):873-83.
12. Sawyer SL, Tian L, Kähkönen M, Schwartzentruber J, Kircher M, University of Washington Centre for Mendelian Genomics, et al. Biallelic mutations in BRCA1 cause a new Fanconi anemia subtype. Cancer Discov, 2015;5(2):135-42.

13. Vallon-Christersson J, Cayanan C, Haraldsson K, Loman N, Bergthorsson JT, Brøndum-Nielsen $\mathrm{K}$, et al. Functional analysis of BRCA1 C-terminal missense mutations identified in breast and ovarian cancer families. Hum Mol Genet, 2001;10(4):353-60

14. Orban TI, Olah E. Expression profiles of BRCA1 splice variants in asynchronous and in G1/S synchronized tumor cell lines. Biochem Biophys Res Commun. 2001;280(1):32-8.

15. Orban Tl, Olah E. Emerging roles of BRCA1 alternative splicing. Mol Pathol, 2003;56(4):191-7.

16. Rosenthal ET, Bowles KR, Pruss D, van Kan A, Vail PJ, McElroy H, et al. Exceptions to the rule: Case studies in the prediction of pathogenicity for genetic variants in hereditary cancer genes. Clin Genet. 2015. doi:10.1111/ cge.12560

17. Whiley PJ, de la Hoya M, Thomassen M, Becker A, Brandão R, Pedersen IS, et al. Comparison of mRNA splicing assay protocols across multiple laboratories: Recommendations for best practice in standardized clinical testing. Mol Diag Genet. 2014;60(2):341-352

18. Tesoriero AA, Wong EM, Jenkins MA, Hopper JL, Brown MA, ChenevixTrench $\mathrm{G}$, et al. Molecular characterization and cancer risk associated with BRCA1 and BRCA2 splice site variants identified in multiple-case breast cancer families. Hum Mutat. 2005;26(5):495.

19. Dosil V, Tosar A, Cañadas C, Pérez-Segura P, Díaz-Rubio E, Caldés T, et al. Alternative splicing and molecular characterization of splice site variants: BRCA1 c.591C>T as a case study. Clin Chem. 2010;56(1):53-61.

\section{Submit your next manuscript to BioMed Central and we will help you at every step:}

- We accept pre-submission inquiries

- Our selector tool helps you to find the most relevant journal

- We provide round the clock customer support

- Convenient online submission

- Thorough peer review

- Inclusion in PubMed and all major indexing services

- Maximum visibility for your research

Submit your manuscript at www.biomedcentral.com/submit 